\title{
Estudio de la aptitud de un material como adición activa al cemento
}

\author{
M. ${ }^{\text {a }}$ PILAR DE LUXAN, Dra. en Ciencias Quimicas \\ M. $^{a}$ ISABEL SANCHEZ DE ROJAS, Leda. en Ciencias Quimicas \\ IETCC/CSIC
}

\section{RES UMEN}

El estudio de la aptitud de un material para su empleo como adición activa al cemento $y$, en definitiva, la valoración de su capacidad puzolánica es un problema que se presenta frecuentemente.

En este trabajo se expone una línea de actuación para abordarlo de un modo práctico y concreto, señalando métodos de posible aplicación, en tanto no exista una normativa al respecto.

\section{$S U M M A R Y$}

The study of the suitability of a material for its use as an active addition to the cement and, really; the evaluation of its puzzolanic capacit! is a problem which is frecuently present.

In this work, a line of behaviour to approach this problem is explained in a practical and definite way; pointing methods of possible application, while there is not a normative about the matter.

\section{INTRODUCCION}

En numerosas ocasiones se plantea la cuestión de si es posible, o no, utilizar un determinado material, para incorporarlo como adición activa en el cemento. Las reiteradas consultas recibidas, centradas sobre este tema, por parte de los usuarios, sobre diferentes materiales, han motivado la realización de este artículo, con la exposición breve y práctica de cómo abordar este problema.

El punto de partida en este planteamiento, habrá de ser la fijación del concepto del término "adición activa" en el cemento, sin entrar en su fundamento, para atender luego al modo de valorar esta actividad en un material concreto.

El concepto de "actividad" o "capacidad de reacción" en este campo es sinónimo de "puzolanicidad". Es decir, que un material se podrá considerar como adición activa, si es capaz de reaccionar con la cal (caso de encontrarse incorporada al cemento, liberada por la base portland en su hidratación), a temperatura ambiente y en presencia de agua, para formar compuestos hidráulicos semejantes a los originados en la hidratación de los constituyentes del clinker portland. Los materiales que presentan esta propiedad, se denominan "puzolanas" en términos generales, aunque en un sentido más amplio se designarán como materiales con propiedades puzolánicas.

Son muchas las clasificaciones que se han realizado con estos materiales (1), (2), (3), ... sin que se haya llegado a una forma definitiva. En España se reconocen como materiales activos: puzolanas de Canarias, Ciudad Real, Gerona, Murcia, ..., y cenizas volantes procedentes de centrales termoeléctricas,...

El tratar de valorar esta actividad, ha provocado la realización de muchos trabajos en la búsqueda del método más adecuado, por falta de un criterio unificado debido a la complejidad del problema. 
A continuación se presenta una vía práctica para seguir, no excluyente de otras, cuando se trate de analizar la aptitud de un material para su aprovechamiento como adición activa o puzolana en el cemento.

\section{ESTUDIO DE UN MATERIAL PARA SU EMPLEO COMO ADICION ACTIVA AL CEMENTO}

\subsection{Valoración de la actividad puzolánica de un material}

Los métodos desarrollados para la valoración de la actividad puzolánica, se pueden reagrupar de la siguiente manera:

\section{METODOS QUE SE BASAN EN EL ESTUDIO DEL MATERIAL}

II. METODOS QUE ENSAYAN MEZCLAS DEL MATERIAL CON CAL

\section{METODOS QUE VALORAN EL CEMENTO CON EL MATERIAL ADICIONADO}

La selección, de entre ellos, de un único ensayo para definir un material, se hace particularmente dificil, si se tiene en cuenta que:

- Por la solà observación del propio material, no es posible, por el momento, señalar de antemano si tiene o no actividad puzolánica. (Con el tiempo, el estudio de la estructura de los materiales que tienen actividad puzolánica, conducirá al conocimiento de las causas que la provocan, y de este modo, a través de su análisis, se prodrá predecir el comportamiento del material).

- Por otra parte, en los métodos de los grupos II y III, se valora el comportamiento del material frente a la cal, bien frente a cal apagada, o a la cal procedente de la hidratación de los minerales del clínker. Estos ensayos reflejan el comportamiento del material bajo una propiedad, y en unas condiciones determinadas.

Por todo ello, será conveniente la aplicación de varios ensayos, para obtener resultados más concluyentes, y buscar el idóneo respecto al fin a que se va a destinar el cemento adicionado.

\section{METODOS QUE SE BASAN EN EL ESTUDIO DEL MATERIAL}

A) El ANALISIS QUIMICO del material abre las siguientes posibilidades:

La composición quimica de las puzolanas varía en un campo muy amplio. Como ejemplo se puede observar el cuadro presentado por el Prof. Dr. J. Calleja, mediante una recopilación bibliográfica (1) (Tabla I), para el análisis químico de las puzolanas naturales, tanto de origen mineral como de origen orgánico:

TABLA I

Composición quimica de los materiales puzolánicos (1)

\begin{tabular}{|c|cccccccccc|}
\hline COMPOSICION & P.P.C. & $\mathrm{SiO}_{2}$ & $\mathrm{Al}_{2} \mathrm{O}_{3}$ & $\mathrm{Fe}_{2} \mathrm{O}_{3}$ & $\mathrm{CaO}$ & $\mathrm{MgO}$ & $\mathrm{SO}_{3}$ & $\mathrm{Na}_{2} \mathrm{O}$ & $\mathrm{K}_{2} \mathrm{O}$ \\
\hline $\begin{array}{c}\text { VALORES } \\
\text { LIMITES } \\
(\%)\end{array}$ & $0-15$ & $42-85$ & $5-20$ & $1-14$ & $0-12$ & $0-11$ & $0-1$ & $0-5$ & $0-5$ \\
\hline
\end{tabular}


- La causa de la actividad puzolánica se ha atribuido al estado metaestable del $\mathrm{SiO}_{2}$ y del $\mathrm{Al}_{2} \mathrm{O}_{3}$ libres que contienen. Por tanto, el contenido, en principio, de estos dos óxidos con carácter ácido, es importante a la hora de valorar un material como puzolana. Por este motivo, en algunos países establecen limitaciones en sus normas al respecto: (4), (5), (6), (7), (8) y (9).

Actualmente en el CEN (Comité Europeo de Normalización), se está estudiando la posibilidad de limitación del contenido en sílice soluble para un material puzolánico, que lleva aparejada la fijación del método correspondiente para su determinación.

Es fundamental conocer, además de la composición química y mineralógica, la morfología de los materiales puzolánicos, ya que es determinante de su actividad.

- El residuo insoluble del material (R.I. = ataque ácido-básico y posterior calcinación), hallado según las especificaciones de la normativa vigente, independiente del significado de este residuo, es un dato necesario, frecuentemente, con vistas a su utilización práctica.

Concretamente, en el caso del Pliego de Prescripciones Técnicas Generales para la Recepción de Cementos (RC-75) (10) se fija:

- en el semento portland con adiciones (cementos PA), una limitación en el R.I. del $4 \%$; si la adición es puzolana el limite se eleva al $8 \%$, y, si es ceniza volante, no se limita;

- en el cemento puzolánico, no hay restricciones respecto al valor del R.I.

En caso de normalizaciones futuras, habrá que tener en cuenta las limitaciones correspondientes.

El residuo insoluble de la muestra anhidra, por si sólo, no es necesariamente un indice de la actividad del material.

- El $M g O$, en las puzolanas, no se encuentra en forma de periclasa sino en un estado físico diferente, generalmente vítreo; por esta razón no es perjudicial desde el punto de vista de la expansión del cemento, y no es necesaria su limitación. En el caso de las cenizas volantes, existen limitaciones en Turquía (5), India (7) y Reino Unido (11).

- El valor del $\mathrm{CaO}$, refleja en las puzolanas, en su mayor parte, el contenido de carbonato cálcico del material; por ello, este valor está ligado al de la pérdida por calcinación. En el caso de las cenizas volantes puede existir $\mathrm{CaO}$ libre.

Por lo general, este valor no se limita en las normas [salvo en Turquía, (5), Alemania (6)], y la expansión producida por la presencia de $\mathrm{CaO}$ libre se detecta en el ensayo correspondiente.

- El valor de la pérdida por calcinación recoge, principalmente, el contenido de carbonato cálcico de la muestra y el de agua combinada que, en ciertas puzolanas es importante, tanto por su cuantía, como por las propiedades que les confiere. Respecto a las cenizas volantes es un índice del contenido de carbón y existen limitaciones en la mayoría de los países: (4), (5), (7), (8), (11), (12), (14) y (15).

- El valor del $\mathrm{SO}_{3}$ de las puzolanas es muy bajo. La limitación de este valor en los cementos portland con adiciones activas y en los cementos puzolánicos en el RC-75, es debido a la proporción de base portland que contienen, asi como a las cenizas volantes incorporadas.

En normas de otros paises (4), (6), (9), (11), (12), (14) y (15), también se limita el contenido de $\mathrm{SO}_{3}$ en las adiciones, por la misma causa de posible expansión por la que se limita en el cemento portland. 
- El contenido de álcalis de la puzolana puede jugar un papel importante en la reacción puzolánica en el equilibrio de la reacción con cal.

En USA (4) y en la India (7), se limita su contenido con el fin de prevenir la reacción áridoálcali. También en Alemania (6).

- Algunos países consideran limitaciones en cuanto al contenido de $\mathrm{Cl}^{-},(6)$, (12) y (13).

B) Bajo un punto de vista práctico, sin atender a estudios estructurales, la aplicación de TECNICAS INSTRUMENTALES al estudio del material, podria permitir disponer de patrones adecuados para cada material, y utilizarlos cuando se desee obtener una posterior identificación del mismo; considerando siempre las posibles variaciones que, por su origen, podrá tener dicho material.

C) Respecto a sus REQUERIMIENTOS FISICOS, la propiedad más importante que hay que destacar es la finura [distribución del tamaño de partículas, superficie específica, (6), ...].

Se dan limitaciones sobre el residuo máximo en el tamiz de 40-45 $\mu \mathrm{m}(4),(8),(11),(12),(14) \ldots$

La finura es un factor primordial a considerar en la valoración de la actividad puzolánica de un material.

\section{METODOS QUE ENSAYAN MEZCLAS DEL MATERIAL CON CAL}

Estos métodos entrañan el propio concepto de puzolanicidad.

En el Instituto Eduardo Torroja, a través de la experiencia, se ha llegado a adoptar el siguiente ensayo acelerado para valorar la actividad puzolánica de un determinado material:

\section{A) ENSAYO ACELERADO PARA VALORAR LA ACTIVIDAD PUZOLANICA DE UN MATERIAL}

Este ensayo se basa en la valoración de la reacción puzolana-cal, mediante el ensayo mecánicoresistente a flexotracción y compresión de morteros, tratados al vapor en unas condiciones prefijadas.

\section{a) Materiales utilizados. Características}

El material estudiado se muele a una finura concreta. La elección del grado de finura se realiza atendiendo, principalmente, a las siguientes consideraciones:

- si se desea conocer la actividad del material para un empleo posterior determinado ya conocido, se ensayará en las mismas condiciones de molienda que caracterizarán su utilización en la práctica;

- si se desea conocer la actividad del material en sí misma, la finura elegida oscilará entre 5.000 y $6.000 \mathrm{~cm}^{2} / \mathrm{g}$. u. Blaine, designada como óptima en anteriores estudios de investigación (16), y considerando las limitaciones del ensayo de determinación de la superficie especifica;

- si se desea comparar la actividad puzolánica de varios materiales, los diferentes ensayos se deberán realizar siempre a finura constante. 
La molienda requiere el secado previo de la muestra a $105 \pm 5^{\circ} \mathrm{C}$ en estufa hasta peso constante. El material pasará a través del tamiz de 4.900 mallas $/ \mathrm{cm}^{2}$.

En cualquier caso, la finura es decisiva en la actividad puzolánica de un material.

La cal apagada empleada, es un hidróxido cálcico, con una riqueza superior al $90 \%$ de $\mathrm{Ca}(\mathrm{OH})_{2}$ en peso, y con un contenido de $\mathrm{CO}_{2}$ inferior al $7 \%$.

Se obtiene a partir de cal viva, hidratándola en autoclave.

Para su utilización se seca a $105 \pm 5^{\circ} \mathrm{C}$ y se muele hasta que no se obtenga residuo por el tamiz de 4.900 mallas $/ \mathrm{cm}^{2}$.

La arena silícea utilizada es la normalizada en el RC-75 (10), para el ensayo de mortero normal de cemento.

La relación agua/conglomerante es 0,50 .

\section{b) Procedimiento}

Las probetas se fabrican de acuerdo con las especificaciones para mortero normal de cemento [apartado 7.6.1. del RC-75, (10)]. Las probetas son, pues, prismas de $4 \times 4 \times 16 \mathrm{~cm}$.

El mortero se fabrica con $500 \pm 2 \mathrm{~g}$ de conglomerante puzolana-cal, $1.500 \pm 5 \mathrm{~g}$ de arena normalizada y $250 \pm 1 \mathrm{cc}$ de agua.

El conglomerante puzolana-cal es una mezcla de 3 partes del material ensayado como puzolana y 1 parte de cal (en peso). (NOTA).

Conservación de las probetas:

Las probetas ya fabricadas se conservarán dentro de los moldes durante $48 \pm 2$ horas, en una cámara a $21 \pm 2^{\circ} \mathrm{C}$ y una humedad relativa superior al $90-95 \%$.

Una vez desmoldadas, se colocan en un ambiente de vapor producido por agua mantenida a $50 \pm 5^{\circ} \mathrm{C}$, en un compartimento estanco durante $72 \pm 3$ horas. Al cabo de este tiempo, se apaga el baño y se dejan enfriar las probetas gradualmente en su interior hasta temperatura ambiente; esto ocurre en $5 \pm 1 / 2$ horas.

El baño requerido en este ensayo se ha diseñado en el Instituto Eduardo Torroja.

Seguidamente se procede a la rotura de las probetas a flexión y a compresión, de forma análoga a como se procede con un ensayo de cemento, de acuerdo con las especificaciones de la norma (10).

c) Resultados

Las resistencias se expresan en $\mathrm{MPa}\left(\mathrm{N} / \mathrm{mm}^{2}\right)$.

NOTA: Si el mortero quedase muy seco, se podria incrementar el agua a 270-300 cc, para conseguir una consistencia adecuada para una buena compactación, pero siempre se debe cuidar mantener la cifra lo más constante posible y, en caso de variación, tenerlo en cuenta a la hora de valorar los resultados. 
Se deberán romper al menos 6 prismas, y se considerará que la resistencia del mortero tanto a flexión como a compresión, viene expresada por el valor medio de los resultados obtenidos, desechando los valores discrepantes (apartado 7.6.1.10 del RC-75).

La valoración de la actividad puzolánica mediante el ensayo aquí descrito, se ha tomado como método habitual en el Instituto. Por ello, son numerosos los resultados que de él se conocen, aplicado a una gran variedad de muestras.

Se ha hecho una selección de algunos valores, para ofrecer una perspectiva amplia de aplicación en cuanto al origen de las muestras; así en la Tabla II, se recogen resultados obtenidos, distribuyendo los materiales en cuatro grupos: cenizas volantes, materiales puzolánicos, rocas siliceas y materiales no puzolánicos, y escogiendo en cada uno de ellos cuatro muestras distintas que proporcionen valores de resistencia mecánica a compresión en un intervalo suficientemente amplio.

Se incluyen también en la Tabla los valores de resistencia a flexión, así como la relación agua/conglomerante, con que se han fabricado las probetas.

En la figura 1 se representan gráficamente los valores de la resistencia mecánica que figura en la Tabla II.

\section{d) Discusión de resultados}

El solo hecho de que una probeta mantenga su forma prismática y no se desmorone, es ya un indice de que el material ha combinado con la cal y de que ha formado compuestos hidráulicos.

Las resistencias a flexotracción normalmente son muy bajas. En los materiales no puzolánicos, es frecuente que el valor sea cero (Tabla II, fig. 1).

Los valores de la resistencia a compresión son más representativos. Los resultados son una expresión de la reacción puzolánica que ha tenido lugar. En la tabla II se observa cómo en cada grupo, los valores encontrados pueden ser muy dispersos entre las distintas muestras.

Sobre este método que se presenta, se pueden hacer las siguientes consideraciones:

- El método permite la evaluación de la capacidad de reacción de un material con la cal.

- Este método tiene validez como requerimiento de aceptación de un material.

- Al ser realizado en parte a $50 \pm 5^{\circ} \mathrm{C}$, es capaz de ofrecer resultados a corto plazo; no obstante, debido a este tratamiento térmico, puede que no represente el comportamiento en la realidad en el hormigón.

- No permite conocer la evolución de la reacción puzolánica del material con el tiempo.

- Ofrece la posibilidad de comparar la reacción puzolana-cal de varios materiales semejantes entre si, es decir, no seria real la comparación entre un material con propiedades hidráulicas per se, de otro que no las tenga.

- La fijación de un valor de resistencia mecánica a compresión que delimite entre material activo y no activo, no puede ser estricta.

Como orientación se podrian considerar los valores para la resistencia mecánica a compresión dados en la Tabla III, aun cuando habrán también de tenerse en cuenta los resultados obtenidos de resistencia a flexotracción. 
T ABLA II

Ensayo acelerado de actividad puzolánica IET

\begin{tabular}{|c|c|c|c|c|}
\hline \multirow{2}{*}{ MUESTRA } & № & $\begin{array}{c}\text { RESISTENCIA A } \\
\text { FLEXOTRACCION } \\
\text { (MPa) }\end{array}$ & $\begin{array}{c}\text { RESISTENCIA A } \\
\text { COMPRESION } \\
\text { (MPa) }\end{array}$ & $\begin{array}{c}\text { RELACION } \\
a / c\end{array}$ \\
\hline \multirow{2}{*}{ CENIZAS } & 1 & 0,4 & 1,1 & 0,50 \\
& 2 & 1,4 & 5,1 & 0,50 \\
& 3 & 1,5 & 6,8 & 0,50 \\
MOLANTES & 4 & 1,7 & 9,8 & 0,50 \\
\hline PUZOLANICOS & 3 & 0,4 & 3,3 & 0,50 \\
& 4 & 1,0 & 5,7 & 0,50 \\
& 1 & 1,5 & 8,7 & 0,50 \\
ROCAS & 2 & 0,4 & 10,4 & 0,54 \\
SILICEAS & 3 & 0,4 & 2,5 & 0,50 \\
& 4 & 0,4 & 4,6 & 0,50 \\
& 1 & 2,1 & 5,3 & 0,50 \\
\hline \multirow{3}{*}{ MATERIALES NO } & 2 & 0,3 & 6,2 & 0,50 \\
\hline PUZOLANICOS & 3 & 0,0 & 1,0 & 0,54 \\
& 4 & 0,0 & 1,5 & 0,50 \\
\hline
\end{tabular}

T AB LA III

Clasificación propuesta sobre la actividad puzolánica de un material en función de la resistencia a compresión en el ensayo acelerado IET

\begin{tabular}{|c|c|}
\hline $\begin{array}{c}\text { RESISTENCIA A } \\
\text { COMPRESION (MPa) }\end{array}$ & $\begin{array}{c}\text { CLASIFICACION PROPUESTA } \\
\text { PARA EL MATERIAL }\end{array}$ \\
\hline Menos de 2 & Poco activo \\
Entre $2-4$ & Actividad baja \\
Entre $4-7$ & Actividad media \\
Entre $7-10$ & Actividad alta \\
Superior a 10 & Muy activo \\
\hline
\end{tabular}

MATERIALES DE CONSTRUCCION. VoI. 35, n.* 200, octubre/noviembre/diciembre 1985 


\section{ENSAYO DE ACTIVIDAD PUZOLANICA}

\section{I-RESISTENCIA A FLEXOTRACCION}

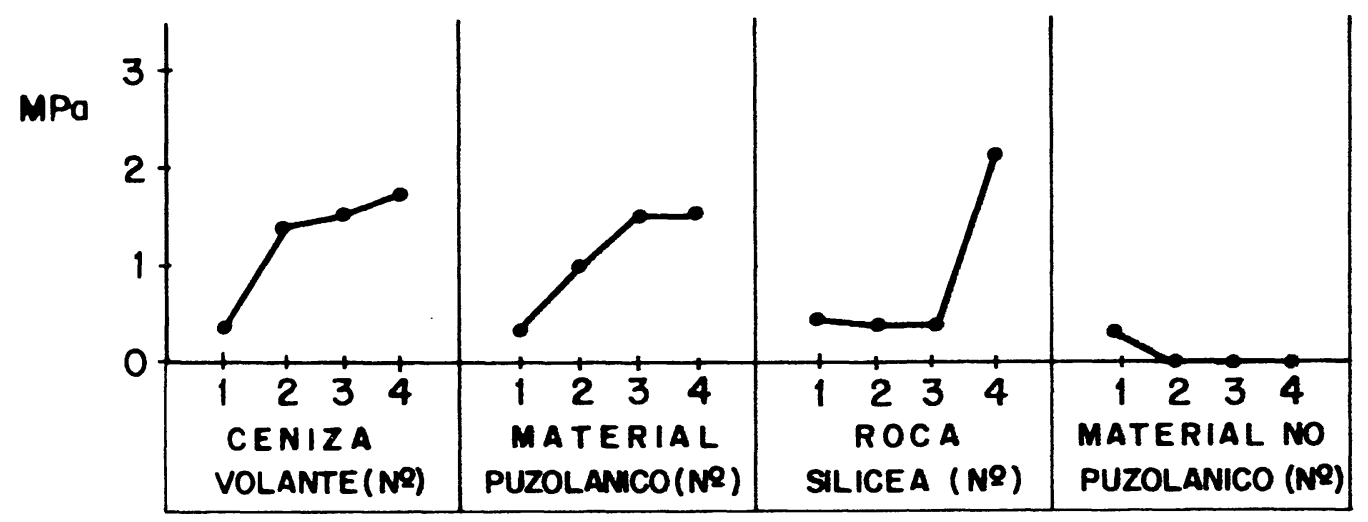

II- RESISTENCIA A COMPRESION

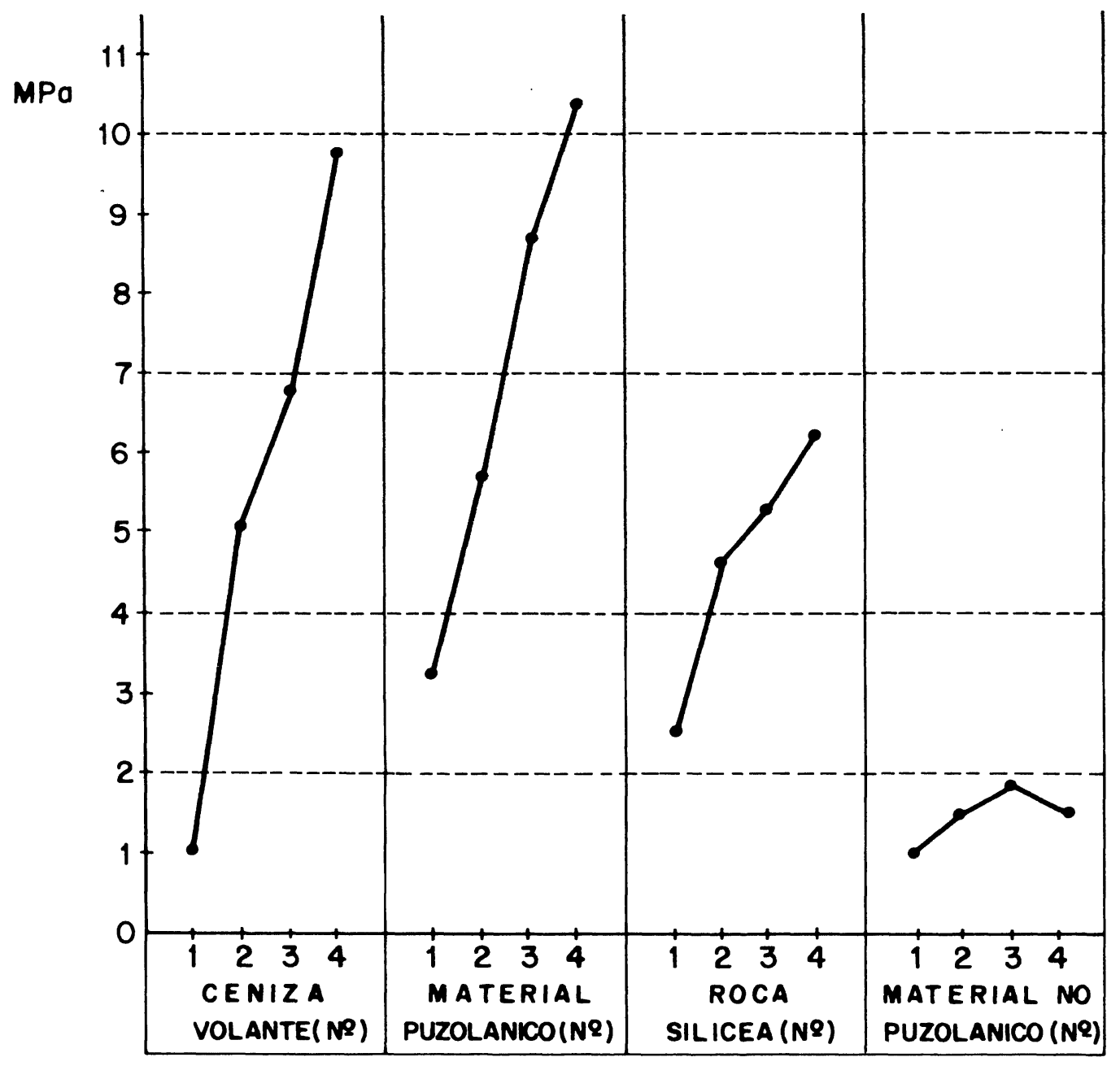

Fig. 1 


\section{B) OTROS METODOS}

Existen otros métodos recogidos en normas extranjeras que valoran la actividad puzolánica de un material en sus mezclas con cal. En España no existe, de momento, normativa al respecto.

Entre estos paises se encuentra: USA (4) y (17), Portugal (18), Alemania (6), India (19),... cuyo indice de actividad puzolánica está basado en ensayos mecánico-resistentes. Otras líneas posibles son valoraciones de la cal en sistemas: material en estudio-cal-agua.

\section{METODOS QUE VALORAN EL CEMENTO CON EL MATERIAL ADICIONADO}

La aplicación de estos métodos tiene sentido cuando se realizan sobre mezclas: cementomaterial, en las que el cemento será el empleado para fabricar más tarde el cemento mixto, ya que el comportamiento de un material varía frente a cada tipo de clínker (20).

En este grupo se puede hacer la siguiente subdivisión, respecto a mezclas cemento/material en estudio:

\section{A) METODOS BASADOS EN ENSAYOS MECANICO-RESISTENTES}

De entre ellos se pueden citar:

- Métodos que prescriben una resistencia mecánica de la mezcla cemento/material en estudio, como un porcentaje de la que alcanzaría el cemento base utilizado sin la adición a una edad prefijada: (4) y (17), (12), (15), (20),...

- Métodos que establecen relaciones en las resistencias mecánicas, tanto en cuanto a variaciones en las proporciones de mezcla, como en las condiciones de curado de las probetas, (11).

\section{B) METODOS BASADOS EN ENSAYOS POR VIA QUIMICA}

El más destacado, por su difusión, es el ensayo de puzolanicidad, cuya revisión y critica fue motivo de un trabajo anterior: (21) y (22). Como alli se citaba, este ensayo se encuentra incluido en las normas de muchos países, y últimamente lo ha recogido también el Comite Europeo de Normalización (CEN) en su normativa.

Las prescripciones de este ensayo son relativas a cementos puzolánicos $y$, en general, a cementos con alto contenido en material activo.

\subsection{Valoración de otras propiedades del material para su uso como adición activa al cemento}

Además de la valoración de la actividad puzolánica del material en estudio, que lo hará apto para su denominación como adición activa, es necesario que el cemento resultante reúna las caracteristicas adecuadas que permitan su fabricación y aplicación en la práctica.

Para la elaboración del cemento mixto, se fijará el porcentaje óptimo de puzolana de acuerdo con las siguientes consideraciones:

- Proporción cemento/material activo en el cemento mixto; finura óptima de ambos o en su conjunto. 
- Aplicación concreta del cemento obtenido. De ella se deducirá qué propiedad del cemento es más fundamental (durabilidad y resistencia química, resistencia mecánica a corto o largo plazo,...).

- Finalidad del cemento, para encuadrarlo en los distintos tipos de cementos con adiciones, según sea la normativa vigente.

- Propiedades específicas que confiere el material puzolánico adicionado al cemento, y que dependen fundamentalmente de su origen. Así, si la puzolana es de tipo tobáceo, más o menos plástica, se eleva siempre el contenido de agua normal; mientras que las puzolanas de aspecto escoriáceo, reducen las necesidades de agua de amasado, son fluidificantes y aumentan, por tanto, el poder resistente de los hormigones con ellos fabricados, al emplear una relación agua/conglomerante más baja, (16).

- Si el material estudiado es nuevo en cuanto a su aplicación en el campo de los conglomerantes hidráulicos, se estudiará además, en su caso, la existencia de algún elemento o caracteristica particularmente nociva.

\section{CONSIDERACIONES FINALES}

Como resumen, en la práctica, y en el momento presente, atendiendo a la normativa vigente, al estudiar la posibilidad de emplear un material como adición activa al cemento, se podrá proceder realizando los siguientes pasos, expuestos esquemáticamente:

\section{1) Estudio del material}

Determinación en el material de la composición química y mineralógica, así como su estado morfológico. Por sí solo, el análisis químico no es suficiente.

Si se trata de destinar el material puzolánico a la fabricación de cemento portland con adiciones activas (PA), es necesario conocer el valor del residuo insoluble (R.I.), y proceder de acuerdo con el RC-75 (10), en tanto siga vigente.

\section{2) Estudio de la actividad puzolánica del material}

El ensayo acelerado de valoración de la actividad puzolánica, descrito en el apartado 2.1., método II A), permite establecer un criterio sobre la capacidad de reacción con cal del material.

\section{3) Estudio de la mezcla: cemento/material puzolánico}

El ensayo de puzolanicidad que se establece en la normativa RC-75 (10), es aplicable a los cementos con contenido de puzolana alto, y concretamente para los cementos puzolánicos.

Este ensayo se realizará sobre el cemento con puzolana ya caracterizado (respecto a proporción, finura,...) y que será el fabricado posteriormente. Los resultados obtenidos serán un reflejo de la actividad puzolánica del material en el cemento mixto.

Por otra parte, se deberá hacer un estudio más completo del cemento preparado. Se observará, en primer lugar, si se atiene a las limitaciones de las normas vigentes y, en segundo lugar, si posec, tanto las caracteristicas y propiedades del cemento, ajenas a dichas especificaciones, pero necesarias para su uso, como son por ejemplo: poder de retención de agua, manejabilidad.... como aquellas que se deseen en su aplicación particular: resistencia química,... 


\section{B I B L I O G R A F I A}

(1) CAlleJA, J.: “Las puzolanas”. II Premio Luxán. Ión, 340, 341, 343 y 344, (1968).

(2) SERSALE, R.: "Structure et caractérisation des pouzzolanes et des cendres volantes", VII Int. Congr. Chem. Cem. vol. I, IV 1-21, Paris 1980.

(3) MASSAZZA, F.: "Chemistry of pozzolanic additions and mixed cements", VI Int. Congr. Chem. Cem. (moscow); Il Cemento 1, 3-38 (1976).

(4) American Society for Testing Materials. "Standard Specification for Fly Ash and Raw or Calcined Natural Pozzolan for use as a Mineral Admixture in Portland Cement Concrete". ASTM C 618-83.

(5) Turkish Standards Institution. "Fly Ashes Suitable for Mixing with Portland Cement Concrete". TS 639-1968.

(6) Deutsche Normen. “Trass. Anforderungen. Prüfung”. DIN 51 043. 1979.

(7) Indian Standards Institution: "Standard Specification for Fly Ash. (Part 2: For Use as Admixture for Concrete)". IS: 3812 (Part 2) - 1966.

(8) Japanese Industrial Standard. “Fly Ash”. JIS A 6201 - 1977.

(9) GOST. "Binder Active Mineral Additives”. GOST 6269 - 63.

(10) Pliego de Prescripciones Técnicas Generales para la Recepción de Cementos. (RC-75). B.O.E. 28-29 agosto 1974.

(11) British Standards Institution. "Pulverized-Fuel Ash. Part 1: Specification for pulverized-fuel ash for use as a cementitious component in structural concrete". BS 3892:1982.

(12) Deutsche INormen. DIN 1045-1980. Richtlinie für die Erteilung von Prüfzeichen für Steinkohlenflugasche als Betonzusatzstoff nach DIN 1045 Bautechnik (11), 39, (1980).

(13) Österreichisches Normungsinstitut. "Draft Specification for Fly Ash for Concrete Production". B3320-1981.

(14) Standards Association of Australia. "Fly Ash for use in Concrete". AS 1129 y 1130 - 1971.

(15) Canadian Standards Association. "Supplementary Cementing Materials and their use in Concrete Construction". CAN3 - A23.5 - M82.

(16) BARBA, C.: "Contribución al estudio de los materiales puzolánicos". Tesis Doctoral. Universidad de Madrid. (1968).

(17) American Society for Testing Materials. "Standard Methods of Sampling and Testing Fly Ash or Natural Pozzolans for Use as a Mineral Admixture in Portland Cement Concrete" ASTM C311-77.

(18) Pliego de Condiciones para el Suministro y Recepción de Puzolanas. Diario do Governo 1 junio 1960.

(19) Indian Standards Institution. "Standard Specification for Burnt Clay Pozzolana”. IS: 1344 - 1968.

(20) DE LUXAN, M.: “Comentarios al Pliego General de Condiciones para la Recepción de Conglomerantes Hidráulicos en Obras de Carácter Oficial", Cemento-Hormigón, no. 364, 377-385, (1964).

(21) DE LUXAN, M. P., SORIA, F.: "Study and Critical Review of the Pozzolanicity Test", Cement and Concrete Research, n.o 5, vol. 5, 461-480, (1975).

(22) DE LUXAN, M. P., SORIA, F.: “A Reply to Dr. Bensted's Discussion on "Study and Critical Review of the Pozzolanicity Test", Cement and Concrete Research, vol. 7, 463-464, (1977). 